\title{
DNA methylation status of NKX2-5, GATA4 and HAND1 in patients with tetralogy of fallot
}

\author{
Wei Sheng ${ }^{1,3}$, Yanyan Qian², Huijun Wang ${ }^{1,3}$, Xiaojing Ma ${ }^{1,3}$, Ping Zhang ${ }^{3}$, Lianwei Diao ${ }^{5}$, Quan An ${ }^{5}$, Long Chen ${ }^{4}$,
} Duan $\mathrm{Ma}^{2,3^{*}}$ and Guoying Huang ${ }^{1,3^{*}}$

\begin{abstract}
Background: NKX2-5, GATA4 and HAND1 are essential for heart development, however, little is known regarding their epigenetic regulation in the pathogenesis of tetralogy of fallot (TOF).

Methods: Methylation levels were measured in three regions of NKX2-5 (M1: -1596 bp -1374 bp, M2: -159 bp $217 \mathrm{bp}$ and M3: $1058 \mathrm{bp} \sim 1524 \mathrm{bp}$ ), one region of GATA4 (M: -392 bp $107 \mathrm{bp}$ ) and three regions of HAND1 (M1: -887 bp -414 bp, M2: -436 bp 2 bp and M3: 37 bp 398 bp) using the Sequenom MassARRAY platform. QRT-PCR was used to analyze NKX2-5 and HAND1 mRNA levels in the right ventricular myocardium of TOF patients.

Results: TOF patients had a significantly higher NKX2-5_M3 median methylation level than controls (41.65\% vs. 22.18\%; $p=0.0074$; interquartile range [IQR]: $30.46 \%-53.35 \%, \mathrm{~N}=30$ and $20.07 \%-24.31 \%, \mathrm{~N}=5$; respectively). The HAND1_M1 median methylation level was also significantly higher in TOF patients than controls (30.05\% vs. 17.54\%; $p=0.0054$; IQR: $20.77 \%-40.89 \%, N=30$ and IQR: 14.69\%-20.64\%; $N=6$; respectively). The methylation statuses of NKX2-5_M1, NKX2-5_M2, GATA4_M, HAND1_M2 or HAND1_M3 were not significantly different in TOF patients compared to controls. The methylation values for NKX2-5_M3 were negatively correlated with mRNA levels $(r=-0.463, p=0.010, N=30)$ and there was a significant association between HAND1_M1 methylation status and mRNA levels $(r=-0.524, p=0.003, N=30)$ in TOF patients.
\end{abstract}

Conclusions: Aberrant methylation statuses of the NKX2-5 gene body and HAND1 promoter regions are associated with the regulation of gene transcription in TOF patients and may play an important role in the pathogenesis of TOF.

Keywords: DNA methylation, NKX2-5, GATA4 and HAND1 genes, Tetralogy of fallot

\section{Background}

Tetralogy of fallot (TOF) is a cyanotic, congenital cardiac defect that is caused by improper development of the right side of the heart [1]. TOF occurs in 3.6 of every 10,000 live births and accounts for $10 \%$ of all congenital heart defects (CHD) [2]. TOF is a complex heart condition that is characterized by a malalignment of the conal septum, which leads to a rightward deviation of the aorta and results in a large ventricular septal defect, along with varying degrees of right ventricular outflow

\footnotetext{
* Correspondence: duanma@yahoo.cn; gyhuang@shmu.edu.cn

${ }^{2}$ Key Laboratory of Molecular Medicine, Ministry of Education, Department of Biochemistry and Molecular Biology, Institutes of Biomedical Sciences, Shanghai Medical College, Fudan University, Shanghai 20003, China

'Children Hospital of Fudan University, Shanghai 201102, China

Full list of author information is available at the end of the article
}

tract narrowing [3]. Although treatment has advanced dramatically over the past few decades, the exact etiology of TOF is unknown. There are still some TOF patients $(0.5 \%$ to $6 \%)$ that suffer sudden cardiac death, despite undergoing treatment [4]. Genetic studies have identified numerous genes that are responsible for inherited and sporadic congenital heart diseases. Most of these, including NKX2-5, GATA4 and HAND1, encode transcription factors that regulate specific phases of heart development [5]. Mutations in NKX2-5, the gene that regulates cardiac development, have been identified in patients with TOF [6,7]. GATA4 is thought to regulate cardiac gene expression and physically interacts with NKX2-5. Mutations in GATA4 may lead to defective interactions with $N K X 2-5$, leading to congenital heart diseases [8]. HAND1 is a basic, helix-loop-helix transcription

\section{Biomed Central}


factor that is essential for mammalian heart development. Mutations in this gene have been reported in patients with ventricular septal defect (VSD) $[9,10]$. Mutations of single genes, including JAG1 [11,12], JAG5 [13], ZFPM2/FOG2 [14] and TBX20 [15], have reportedly been found in TOF patients. However, single gene mutations or absences have only been found in a small percentage of patients and are not considered to be the main cause of TOF [16]. Furthermore, reports of somatic gene mutations in formalin-fixed, paraffin embedded cardiac tissue have not been confirmed in subsequent studies with fresh, frozen cardiac tissue, which consistently demonstrate an absence of somatic gene mutations. In fixed cardiac tissue, formalin can cause random base damage, affecting polymerase chain reaction (PCR) fidelity [17], thereby increasing the risk for poor data quality. Therefore, somatic mutation data obtained in fresh, frozen cardiac tissue may be more accurate and the incidence of single gene mutations is likely lower than is indicated from studies of fixed cardiac tissue. Interestingly, most of the genes above have shown significant changes in expression in the cardiovascular tissue of TOF patients [18]. Epidemiological data has also indicated that environmental influences may play a role in the etiology of TOF [19]. Epigenetic influences must be considered in order to understand the mechanisms that lead gene-environment interactions to cause disease [20]. Currently, the most widely studied epigenetic modification in humans is DNA methylation, which occurs almost exclusively in the context of $\mathrm{CpG}$ dinucleotides that control the transcriptional activity of genes [21]. Aberrant DNA methylation can result in silencing gene expression and functional inactivation [22]. This has been demonstrated in various human diseases, such as glioblastoma [23], chronic lymphocytic leukemia (CLL) [24] and invasive cervical cancer (ICC) [25]. Although mutations in NKX2-5, GATA4 and HAND1 have been found in patients with TOF, little is known about changes in these genes due to DNA methylation.

The goal of the present study was to explore DNA methylation changes in NKX2-5, GATA4 and HAND1 and to examine the epigenetic regulation of these genes in the right ventricular myocardium of TOF patients. These results may offer a deeper understanding of the etiology of this disease and provide important clues for the development of new treatments for TOF.

\section{Methods}

\section{Patients and controls}

TOF cases were obtained from the Children's Hospital of Fudan University, Shanghai, China. Cardiovascular diagnosis was done using echocardiography. All TOF subjects were assessed for 22q11.2 deletions; only TOF patients without the $22 \mathrm{q} 11$ deletion syndrome were included. A total of 30 patients with TOF were studied, including 20 (66.7\%) males and 10 (33.3\%) females, ranging in age from 0.25 to 4.0 years (mean \pm SD: $1.13 \pm$ 0.85 years).

The control group was comprised of autopsy specimens from normal subjects that had died as a result of accidents. Specimens were collected at the Forensic Medicine Department of Fudan University, Shanghai, China. Control samples were chosen in which the time interval between death and autopsy was as short as possible so that any delays before autopsy would be taken into account. The post mortem interval (PMI) for the control samples was no more than 24 hours. Specimens from six age-matched normal controls were obtained, including 4 (66.7\%) males and 2 (33.3\%) females, ranging in age from 0.5 to 4.5 years (mean $\pm \mathrm{SD}$ : $1.73 \pm$ 1.44 years). Characteristics of the study subjects are summarized in Additional file 1: Table S1.

All tissue samples were obtained from the right ventricular myocardium tissues immediately after surgical resection or autopsy and stored in RNAlater ${ }^{\circ}$ (AMBION, Inc., Austin, TX, U.S.) until use to exclude any tissue heterogeneity that may affect methylation results.

This study was approved by the local ethics committee of Fudan University. Written informed consent was obtained from the parents or relatives of all study subjects.

\section{DNA extraction and sodium bisulfite conversion}

A QIA amp DNA Mini Kit (Qiagen, Hilden, Germany) was used to extract genomic DNA, according to the manufacturer's instructions, from the heart tissue samples of TOF patients and controls. The concentration and purity of genomic DNA were measured via absorbance at 260 and $280 \mathrm{~nm}$ using a NanoDrop ${ }^{\mathrm{TM}} 1000$ Spectrophotometer (Thermo Scientific, Wilmington, U.S.). Sodium bisulfite modification of genomic DNA was done, strictly according to manufacturer's instructions, using an EZ DNA Methylation $\mathrm{Kit}^{\mathrm{tm}}$ (Zymo Research, Orange, CA, U.S.). Sequencing results confirmed that more than $99.0 \%$ of cytosine residues were converted. The bisulfite converted DNA was resuspended in $10 \mu \mathrm{l}$ elution buffer and stored at $-80^{\circ} \mathrm{C}$ until the samples were ready for analysis.

\section{Quantitative MassARRAY analysis of gene methylation status}

Quantitative methylation analysis for NKX2-5, GATA4 and $H A N D 1$ was performed based on base-specific cleavage and MALDI-TOF mass spectrometry, as recommended by the manufacturer, using the MassArray EpiTyper (Sequenom, San Diego, CA, U.S.). The Sequenom groups have demonstrated the robustness of this approach for quantifying methylated and unmethylated DNA [26]. The primers used in this study were designed using http://epidesigner.com (Additional file 1: Table S2.). For each reverse primer, an additional T7 promoter tag was added for in vivo transcription and a 10-mer tag was 
added to the forward primer to adjust for the melting temperature differences. All experiments were performed as described previously [27].

The accuracy of the methylation analysis results from the MALDI-TOF MassARRAY (Sequenom) were confirmed by pyrosequencing and bisulfite sequencing PCR (BSP) [28].

\section{RNA extraction and quantitative RT-PCR}

Total RNA was extracted from heart tissue samples using Trizol Reagent (Invitrogen, CA, U.S.), according to the manufacturer's instructions. RNA was reverse-transcribed using a PrimeScript RT reagent Kit with gDNA Eraser (Perfect Real Time, TaKaRa, Otsu, Shiga, Japan) and the integrity of synthesized cDNA was confirmed using glyceraldehyde 3phosphate dehydrogenase (GAPDH) as the endogenous control. Quantitative RT-PCR was performed in a 7900 realtime PCR system using SYBR Premix Ex Taq GC (Perfect Real Time, TaKaRa) using a $10 \mu \mathrm{l}$ reaction volume, containing $5 \mu \mathrm{l}$ SYBR Premix Ex Taq GC, $0.2 \mu \mathrm{M}$ of each primer, $0.2 \mu$ ROX1 Reference Dye and 100 ng cDNA. Reactions were performed in triplicate and analyzed using an ABI 7900 Sequence Detection System (Applied Biosystems). Relative expression levels were calculated according to the standard $2^{-\triangle \Delta C t}$ method using beta-2 microglobulin (B2M) and the GAPDH gene as the endogenous control for normalization. Primer sequences used in the QRT-PCR analysis are listed in Additional file 1: Table S3.

\section{Statistical analysis}

Data were analyzed using GraphPad Prism (version 5.0; GraphPad Software Inc., San Diego, CA, U.S.) and SPSS (version 13.0; SPSS Inc., Chicago, IL, U.S.). The MannWhitney test was performed to evaluate the significance of any differences between the TOF and control groups. Spearman correlation analysis was performed to evaluate the correlations between the methylation statuses and mRNA levels of the NKX2-5 and HAND1 genes. All statistical analysis was 2 -sided and $P<0.05$ was considered statistically significant.

\section{Results}

Methylation levels for NKX2-5, GATA4 and HAND1 genes in TOF patients and controls

The methylation statuses of NKX2-5, GATA4 and HAND1 genes were analyzed using the Sequenom MassARRAY platform. Three amplicons, including three regions, in NKX2-5 (M1: -1596 bp -1374 bp, M2: -159 bp 217 bp and M3: $1058 \mathrm{bp} \sim 1524 \mathrm{bp}$, Figure 1A), one amplicon, including one region, in GATA4 (M: $-392 \mathrm{bp} \sim 107 \mathrm{bp}$, Figure $2 \mathrm{~A}$ ) and three amplicons, including three regions, in HAND1 (M1: -887 bp -414 bp, M2: -436 bp 62 bp and M3: $37 \mathrm{bp} \sim 398 \mathrm{bp}$, Figure 3A) were analyzed in specimens from 10 patients with TOF and 6 age matched controls. Prior to analysis, strict quality control was performed to remove potentially unreliable measurements. The $\mathrm{CpG}$ units that failed to produce data from more than $30 \%$ of samples (unreliable CpG units) and samples missing more than $30 \%$ of the data points (unreliable samples) were discarded [29].

The methylation level of the NKX2-5_M3 amplicon was significantly higher, with a median of $34.12 \%$ (IQR: $32.03 \%-48.86 \%, \mathrm{~N}=10$ ) in TOF patients, compared to a median of $22.18 \%$ in controls (IQR: $20.07 \%-24.31 \%$;

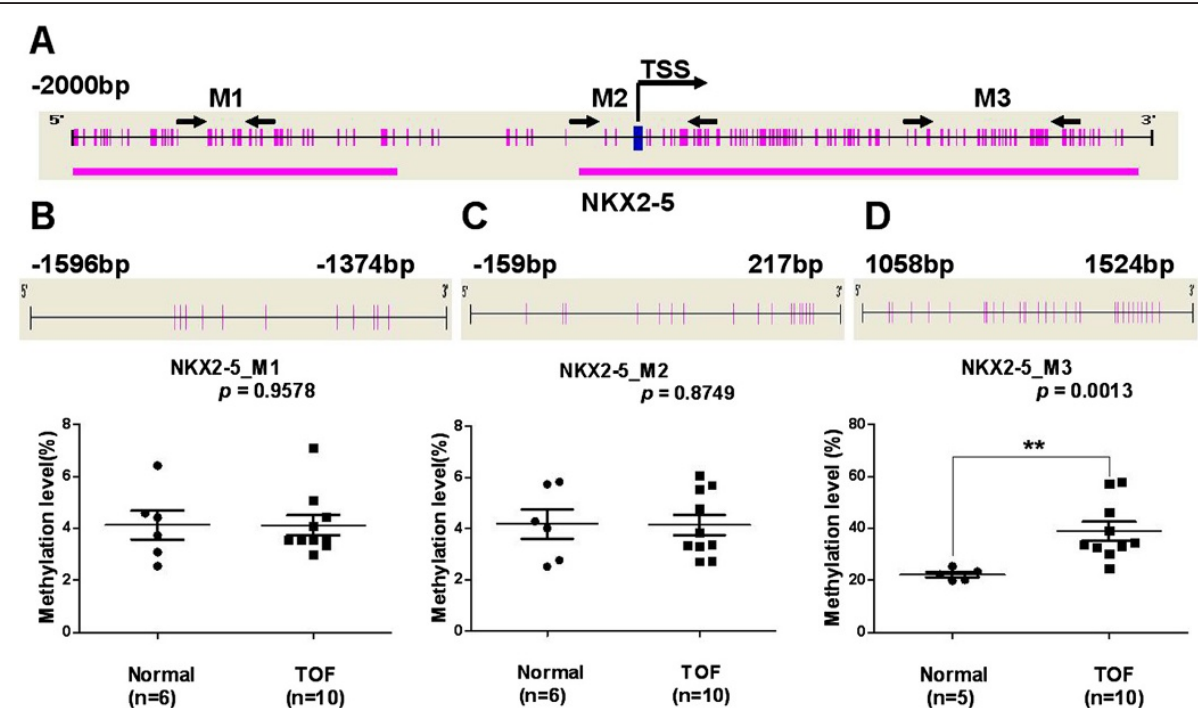

Figure 1 Median methylation levels for NKX2-5 in TOF patients and controls. (A) The schematic represents the distribution of the CpG site and CpG island in the NKX2-5 gene; (B) NKX2-5_M1 median methylation level; (C) NKX2-5_M2 median methylation level; (D) NKX2-5_M3 median methylation level. All of the values represent the median with the interquartile range. TSS, Transcription Start Sites; red vertical line, CpG sites; red thick bars, CpG islands; M,MassARRAY amplicon; The region between arrows, target amplicon. ${ }^{*} p<0.05,{ }^{* *} p<0.01,{ }^{* * *} p<0.001$

(Mann-Whitney test). 


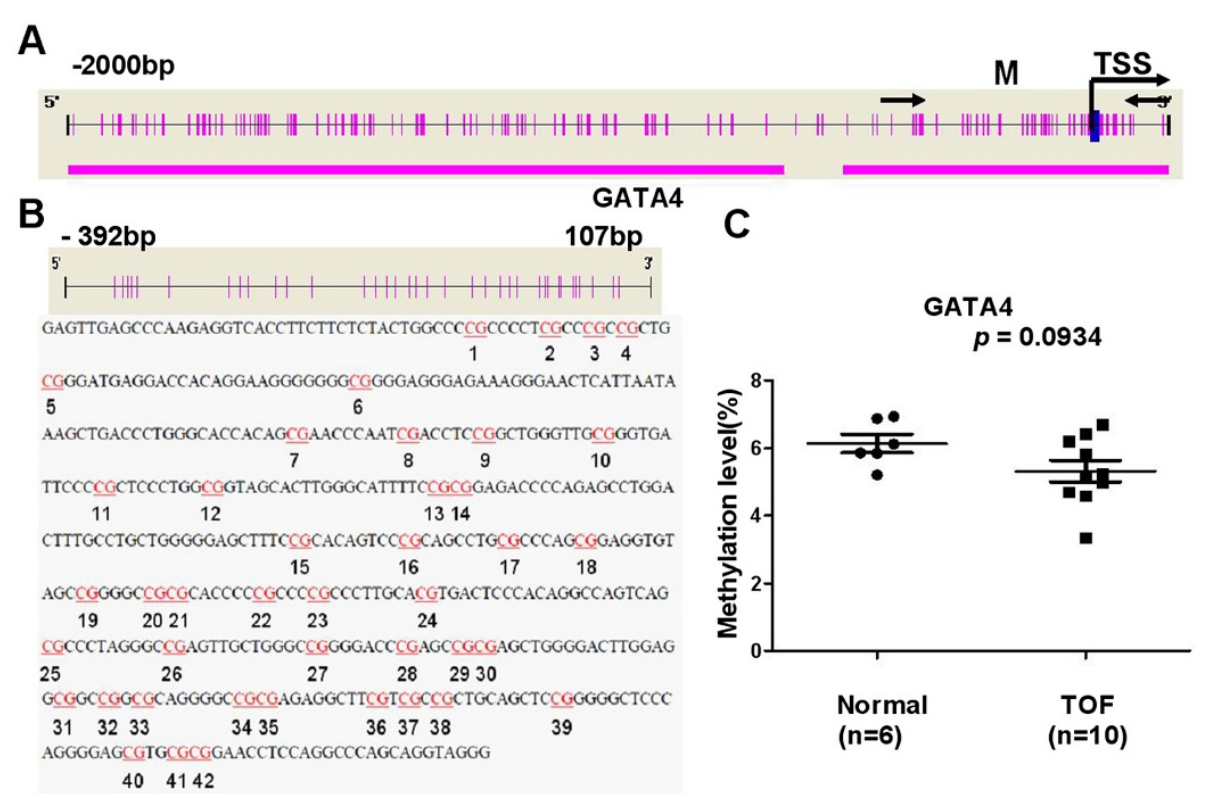

Figure 2 Median methylation levels for GATA4 in TOF patients and controls. (A) The schematic represents the distribution of the CpG site and CpG island in the GATA4 gene; (B) The sequence shown represents a 500 base pair fragment (positions -392 - 107) in the 5'-UTR of GATA4; (C) GATA4_M median methylation level. All of the values represent the median with the interquartile range. TSS, Transcription Start Sites; red vertical line, CpG sites; red thick bars, CpG islands; M,MassARRAY amplicon; The region between arrows, target amplicon. ${ }^{*} p<0.05,{ }^{* *} p<0.01,{ }^{* * *} p<0.001$ (Mann-Whitney test).

$p=0.0013, \mathrm{~N}=5$, one male, aged 1.9 years was not included, Figure 1D). Methylation levels in the NKX25_M1 and NKX2-5_M2 amplicons were not significantly different between the TOF and control subjects (Figure 1B and Figure 1C).
There was no significant difference in the methylation level for GATA4_M when comparing the TOF and control subjects (Figure 2C). The methylation level of HAND1_M1 was significantly higher in TOF subjects, with a median of $30.77 \%$ (IQR: $21.59 \%-51.65 \%, \mathrm{~N}=10$ )

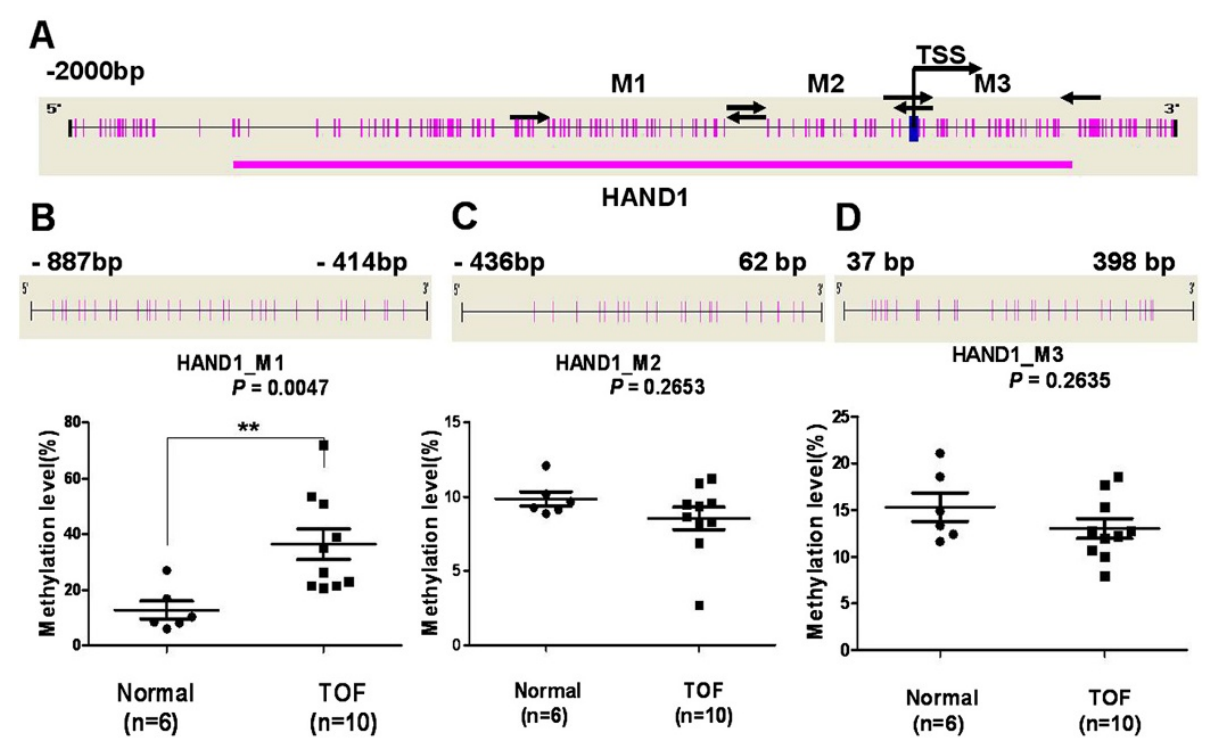

Figure 3 Median methylation levels for HAND1 in TOF patients and controls. (A) The schematic represents the distribution of the CpG site and CpG island in the HAND1 gene; (B) HAND1_M1 median methylation level; (C) HAND1_M2 median methylation level; (D) HAND1_M3 median methylation level. All of the values represent the median with the interquartile range. TSS, Transcription Start Sites; red vertical line, CpG sites; red thick bars, CpG islands; M,MassARRAY amplicon; The region between arrow, target amplicon. ${ }^{*} p<0.05,{ }^{* *} p<0.01,{ }^{* * *} p<0.001$

(Mann-Whitney test). 
compared to $17.54 \%$ (IQR: $14.69 \%-20.64 \% ; p=0.0047$, $\mathrm{N}=6$, Figure $3 \mathrm{~B}$ ) in the control group. No significant difference was observed in the methylation levels for HAND1_M2 and HAND1_M3 when comparing TOF and control subjects (Figure $3 \mathrm{C}$ and Figure 3D, respectively).

Pyrosequence was used to determine the methylation status of NKX2-5_P in 2 TOF patients and in 1 control and BSP was used to measure the methylation level of GATA4_B in 2 TOF patients and in 2 controls to confirm the accuracy of the MALDI-TOF MassARRAY (Sequenom) methylation analysis. NKX2-5_P and GATA4_B overlapped with NKX2-5_M2 and GATA4_M (Figures $4 \mathrm{~A}$ and $5 \mathrm{~A}$, respectively). Primer sequences used are listed in Additional file 1: Table S4.

As shown in Figure 4, the methylation value for NKX2-5_P was less than $10 \%$. This result was similar to that found using the MassARRAY methylation analysis for NKX2-5_M2. Methylation analysis results from BSP for GATA4_B were also consistent with the MassARRAY methylation analysis results for GATA4_M (Figure 5). These data confirmed the accuracy of methylation analysis results from the MALDI-TOF MassARRAY (Sequenom).

Validation of different methylation levels for NKX2-5_M3 and HAND1_M1

The Sequenom MassARRAY platform was used to validate and compare the different methylation levels of NKX2-5_M3 and HAND1_M1 in 20 patients with TOF and 6 age matched controls. As shown in Figure 6A, the methylation level of NKX2-5_M3 was significantly higher in patients with TOF, with a median value of 45.06\% (IQR: 25.93\%-54.36\%, $\mathrm{N}=20$ ) compared to 22.18\% (IQR: $20.07 \%-24.31 \%, p=0.0383, \mathrm{~N}=5$, one male, aged 1.9 years, not included) in controls. Furthermore, patients with TOF also had significantly higher methylation levels for HAND1_M1, with a median value of $30.01 \%$ (IQR: $19.82 \%-39.11 \%, \mathrm{~N}=20$ ) compared to 17.54\% (IQR: $14.69 \%-20.64 \% ; \mathrm{N}=6, p=0.0137$, Figure 6B) in controls.

The NKX2-5_M3 and HAND1_M1 methylation levels in the 20 TOF patients and the initial 10 TOF subjects were combined and compared to those of the control

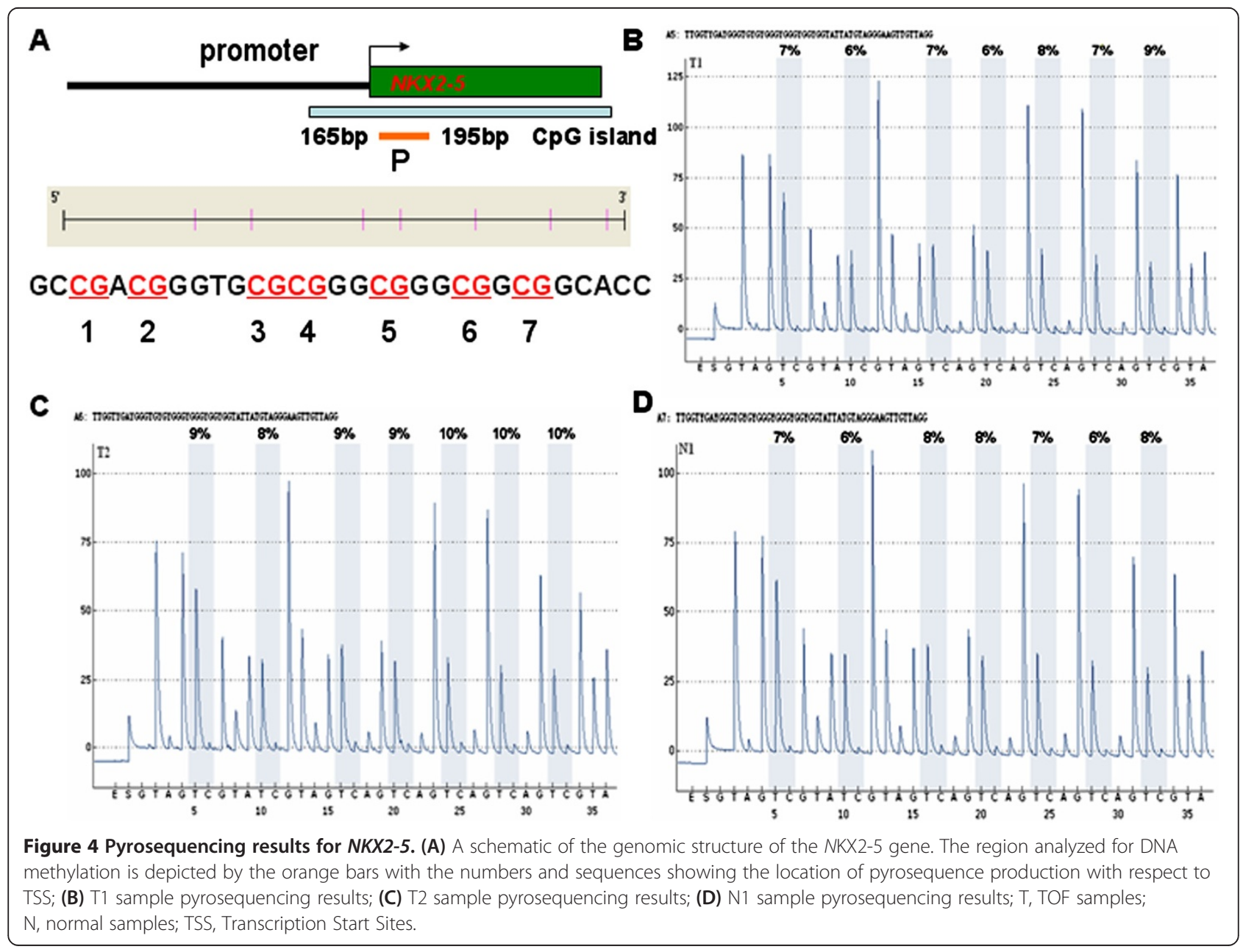




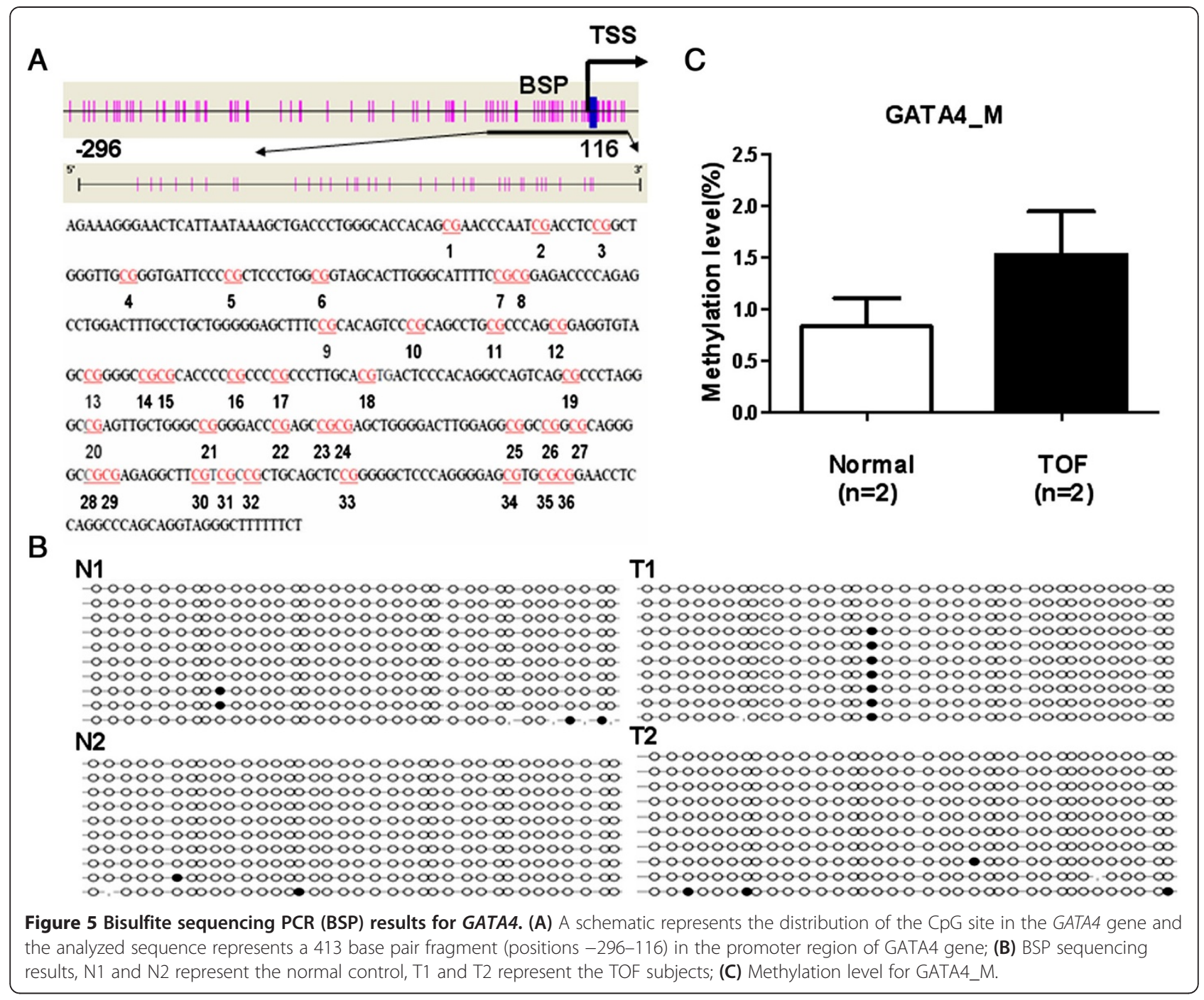

subjects. A significantly higher NKX2-5_M3 methylation level was found in TOF patients, with a median value of 41.65\% (IQR: $30.46 \%-53.35 \%, \quad \mathrm{~N}=30$ ) compared to 22.18\% (IQR: $20.07 \%-24.31 \%, \mathrm{~N}=5$ ) in controls ( $p=$ 0.0074, Figure 6C). The HAND1_M1 methylation level was also significantly higher in TOF patients, with a median value of $30.05 \%$ (IQR: $20.77 \%-40.89 \%, \quad \mathrm{~N}=30$ ) compared to $17.54 \%$ (IQR: $14.69 \%-20.64 \%, \mathrm{~N}=6$ ) in controls $(p=0.0054$, Figure 6D).

Expression levels of NKX2-5 and HAND1 mRNA in patients with TOF and controls

The mRNA expression levels of the NKX2-5 and HAND1 genes were determined by QRT-PCR in 30 TOF patients and 6 controls. The normalized $\mathrm{Ct}$ mean $(\triangle \mathrm{Ct})$, $\triangle \Delta \mathrm{Ct}$ and RQ $\left(2^{-\Delta \Delta \mathrm{Ct}}\right)$ values for NKX2-5 and HAND1 genes in each samples are listed in Additional file 1: Table S5. The RQ value $\left(2^{-\Delta \Delta C t}\right)$ for control 1 was set as 1 and used for normalization for all samples.
As shown in Table 1, NKX2-5 and HAND1 mRNA relative expression levels ( $R Q$ values) were significantly lower in patients with TOF compared with controls $(p<0.05)$.

\section{Association between the methylation statuses and mRNA} levels of NKX2-5 and HAND1

A correlation analysis was done, in TOF patients, to identify any relationship between NKX2-5 and HAND1 methylation status and their respective mRNA levels.

As shown in Figure 7A, a significant association was observed between the methylation status and mRNA level of NKX2-5_M3 $(\mathrm{r}=-0.463, p=0.010, \mathrm{~N}=30)$. Similarly, there was a significant association between the methylation status and mRNA level of the HAND1 gene $(\mathrm{r}=-0.524, p=0.003, \mathrm{~N}=30$, Figure. 7B).

The association between NKX2-5 and HAND1 methylation status and their respective mRNA levels was also analyzed without the initial 10 TOF subjects. 

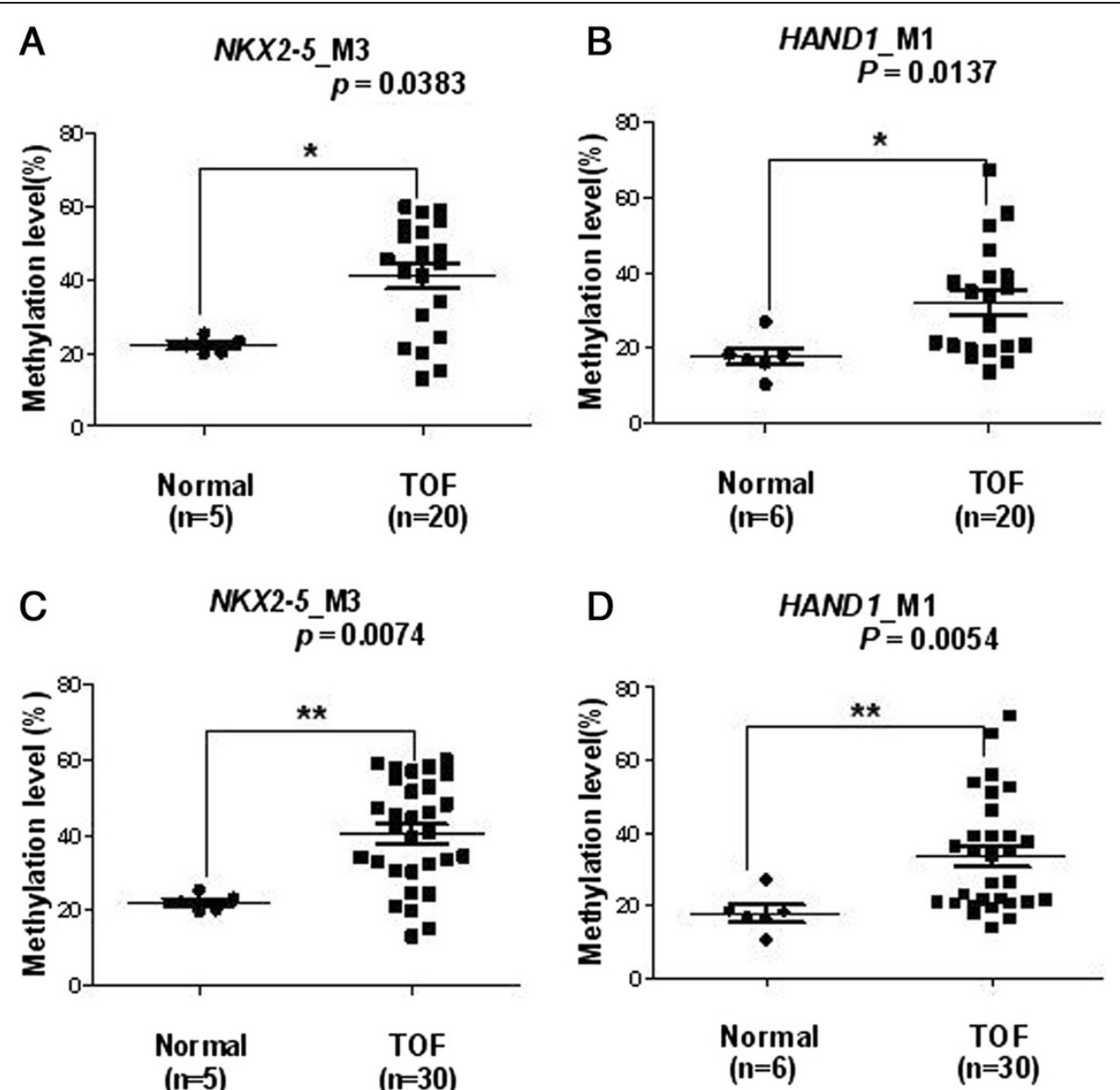

Figure 6 Comparison of median methylation level for NKX2-5_M3 and HAND1_M1 in the control and TOF subjects. (A) Median methylation level for NKX2-5_M3 of 5 control and 20 TOF subjects; (B) Median methylation level for HAND1_M1 of 6 control and 20 TOF subjects; (C) Median methylation level for NKX2-5_M3 of 5 control and 30 TOF subjects; (D) Median methylation level for HAND1_M1 of 6 control and 30 TOF subjects. ${ }^{*} p<0.05,{ }^{* *} p<0.01,{ }^{* * *} p<0.001$ (Mann-Whitney test).

Interestingly, there was a significant correlation found between the NKX2-5_M3 methylation status and mRNA level $(\mathrm{r}=-0.468, p=0.038, \mathrm{~N}=20$, Figure $7 \mathrm{C})$. A significant association was also observed between the methylation status and mRNA level of HAND1 $(\mathrm{r}=-0.478, p=$ 0.033, $\mathrm{N}=20$, Figure 7D).

\section{Discussion}

Epigenetic control mechanisms play key roles in the regulation of tissue homeostasis and disease development. This includes DNA methylation, histone modification, the regulation of mRNA stability, translation by non-coding RNAs and differential RNA splicing [30].
DNA methylation constitutes an important epigenetic regulation mechanism in many eukaryotes and has been extensively studied [31]. In a previous study, decreased LINE-1 methylation levels were found in the cardiac tissue of TOF patients. The lower LINE-1 methylation level may be associated with increased TOF risk [27] and could serve as an indicator of global DNA methylation [32]. In the current study, we initially performed quantitative methylation analysis of NKX2-5, GATA4 and HAND1 genes in the right ventricular myocardium tissues of 10 TOF patients and 6 age matched control subjects using the Sequenom MassARRAY platform and found a significant difference in the methylation levels of

Table 1 Relative mRNA expression levels of NKX2-5 and HAND1 in samples from controls and patients with TOF

\begin{tabular}{|c|c|c|c|}
\hline Gene & Control (RQ value $\left(2^{-\Delta \Delta C t}\right)$ a $:$ mean $\left.\pm S D, n=6\right)$ & TOF (RQ value $\left(2^{-\Delta \Delta C t}\right):$ mean $\left.\pm S D, n=30\right)$ & $P$ value $^{\mathrm{b}}$ \\
\hline NKX2-5 & $9.76 \pm 7.39^{c}$ & $1.48 \pm 3.45$ & 0.0028 \\
\hline HAND1 & $7.55 \pm 5.62$ & $1.20 \pm 0.41$ & 0.0032 \\
\hline
\end{tabular}

${ }^{a}$ The $R Q$ values $\left(2^{-\Delta \Delta \mathrm{ct}}\right)$ for control 1 was set as 1 and used for normalization for all samples; ${ }^{\mathrm{b}}$ Mann-Whitney test was performed; ${ }^{\mathrm{c}}$ " ${ }^{\mathrm{C}}$ " represents the meaning of addition and subtraction. One male, aged 1.9 years, was not included. 


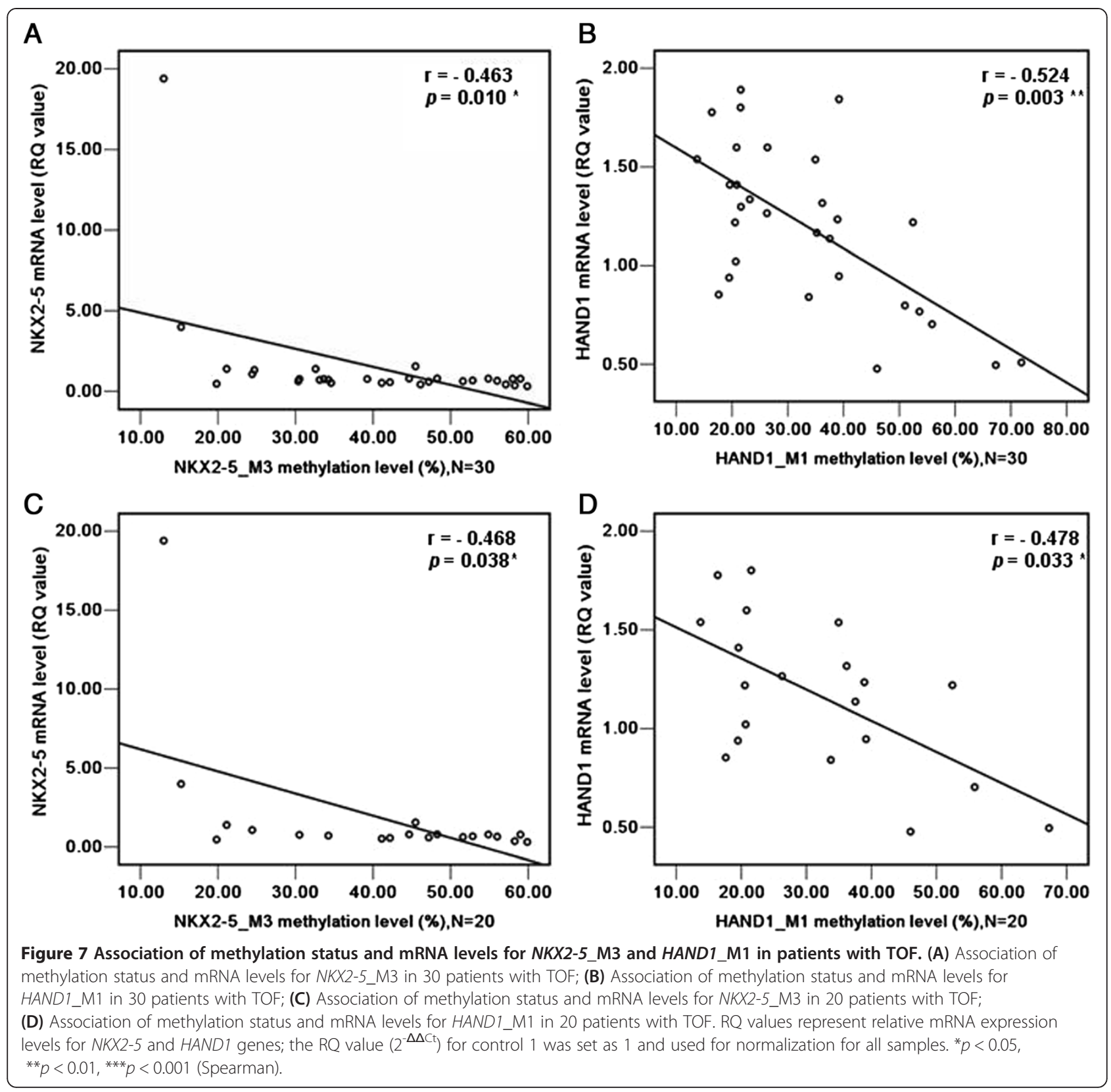

NKX2-5_M3 and HAND1_M1 in TOF patients compared with controls $(p<0.05)$. The methylation values for NKX2-5_M3 and HAND1_M1 were then validated in a larger cohort TOF subjects. The difference in methylation levels for NKX2-5_M3 and HAND1_M1 were compared, between TOF patients and controls, in two independent cohorts. A significant difference was observed in the methylation status of NKX2-5_M3 when comparing the 20 TOF patients to the 6 controls $(p=$ 0.0383). The methylation levels for the promoter region of the HAND1 gene (HAND1_M1) were also significantly different between the 20 TOF patients and the 6 controls $(p=0.0137)$. Interestingly, the significant difference in the methylation levels for NKX2-5_M3 and HAND1_M1 increased when comparing the 30 TOF cases to the 6 controls $(p=0.0074, p=0.0054$, respectively).

The MassARRAY EpiTYPER ${ }^{\circ}$ for quantitative analysis of DNA methylation combines base-specific enzymatic cleavage with MALDI-TOF mass spectrometry. This combination provides a highly accurate, sensitive and high-throughput method for the quantitative analysis of DNA methylation. The EpiTYPER software provides convenient solutions for data analysis and export, however,the Sequenom EpiTYPER has a 5\% technical error rate [33]. Although the robustness of this approach for 
confirming the accuracy of Sequenom methylation analysis results has been demonstrated, we used two alternate methods,pyrosequence and BSP,to test the methylation analysis results of NKX2-5 and GATA4. No apparent differences were detected among these methods. This data supports the accuracy of the Sequenom MassARRAY methylation analysis results in our study.

Increasing evidence suggests that single gene mutations are present in a broad spectrum of genes involved in cardiac structure and function. Factors, such as NKX2-5, GATA4 and HAND1, are among the earliest transcription factors expressed in the developing heart and are crucial in the activation of cardiac-specific genes [34]. Wang et al. sequenced all exons and their boundaries in the NKX2-5, GATA4 and HAND1 genes from non-syndromic TOF children and controls and found no evidence that somatic mutations in NKX2-5, GATA4 and $H A N D 1$ play a role in the pathogenesis of TOF [35]. Although mutations in NKX2-5, GATA4 and HAND1 have been observed in patients with TOF, they are present only in a small percentage of TOF cases and cannot be considered to be the main cause of TOF. Based on these findings, we hypothesized that the aberrant DNA methylation modifies these genes and likely contributes to the development of TOF. In the present study, no significant difference in GATA4 methylation levels were observed between TOF patients and controls, thus the methylation status of GATA4 likely has no influence on its gene expression. Furthermore, we found that mRNA levels of the NKX2-5 and HAND1 genes were significantly lower in TOF patients compared to controls $(p<0.05)$. We also found significant negative correlations between methylation status and mRNA level for NKX2-5 and HAND1 in the 30 TOF cases (Figure 7A and B). Interestingly, the associations between methylation status and mRNA level for NKX2-5 and HAND1 were confirmed in the 20 TOF cases without including the initial $10 \mathrm{TOF}$ subjects (Figure 7C and D). This demonstrates a consistent, significant association via two independent comparisons. These findings indicate that changes in the methylation levels for NKX2-5 and HAND1 may influence gene expression and contribute to the development of TOF.

One limitation of this study was that we were unable to obtain enough complete age-matched samples from TOF patients and healthy controls due to the difficulty of obtaining cardiac tissue samples. Further studies with larger number of subjects or samples are warranted in order to confirm our findings that aberrant methylation contributes to TOF development. Moreover, based only on the current results from clinical samples, we cannot ascertain whether the noted methylation changes occurred after the heart was already formed or after the heart defect already existed. Thus, we cannot determine whether these changes are reflective of disease physiology or related to disease etiology. Related research will be conducted using cell lines or animal models in future studies.

\section{Conclusion}

In the present study, we examined the methylation status of NKX2-5, GATA4 and HAND1 and found significant changes in the methylation levels in the NKX2-5 gene body and the HAND1 gene promoter region. In addition, the aberrant methylation status of NKX2-5 and HAND1 was negatively correlated with their corresponding mRNA expression, indicating that the DNA methylation changes in these two genes contribute to transcription regulation in TOF patients and may play important roles in the pathogenesis of TOF. These findings may provide important clues for the development of novel treatments for TOF and provide a more thorough understanding of the etiology of congenital heart disease.

\section{Additional file}

Additional file 1: Table S1. Anagraphical characteristics of study subjects. Table S2. Primer sequences, position, product length, and CpG unit used for MassArray quantitative methylation analysis. Table S3. Primer sequences and product length for QPT-PCR analysis. Table S4. Primer sets for pyrosequencing and bisulfite sequencing PCR (BSP). Table S5. The values of $\Delta C t$ mean, $\Delta \triangle \mathrm{Ct}$ and RQ for NKX2-5 and HAND1 in controls and TOF subjects.

\section{Competing interests}

The authors declare that they have no competing interests.

\section{Authors' contributions}

SW participated in study concept and design and coordination of the study, helped with the statistical analysis and drafted the manuscript. QY, WH, MX, DL and AQ participated in TOF sample acquisition and helped to draft the manuscript. ZP and $C L$ participated in normal control sample acquisition and helped to draft the manuscript. MD and HG conceived of the study, and participated in its design and coordination and helped to draft the manuscript. All authors read and approved the final manuscript.

\section{Acknowledgements}

This work was supported by grants from the National Natural Sciences Foundation of China (30930096), the National Basic Research Program of China (2010CB529504, 2009CB941704), Shanghai Municipal Commission of Science and Technology Research Project (11JC1401400) and China Postdoctoral Science Foundation (2013 M540324).

\section{Author details}

${ }^{1}$ Children Hospital of Fudan University, Shanghai 201102, China. ${ }^{2}$ Key Laboratory of Molecular Medicine, Ministry of Education, Department of Biochemistry and Molecular Biology, Institutes of Biomedical Sciences, Shanghai Medical College, Fudan University, Shanghai 20003, China.

${ }^{3}$ Shanghai Key Laboratory of Prevention and Intervention of Birth Defects, Shanghai 201102, China. ${ }^{4}$ Department of Forensic Medicine, Fudan University, Shanghai 20003, China. ${ }^{5}$ Wuhe Chinese Medicine Hospital, Wuhe 233300, China.

Received: 18 July 2013 Accepted: 29 October 2013 Published: 1 November 2013 


\section{Reference}

1. Therrien J, Webb G: Clinical update on adults with congenital heart disease. Lancet 2003, 362(9392):1305-1313.

2. Bedard E, McCarthy KP, Dimopoulos K, Giannakoulas G, Gatzoulis MA, Ho SY: Structural abnormalities of the pulmonary trunk in tetralogy of Fallot and potential clinical implications: a morphological study. J Am Coll Cardiol 2009, 54(20):1883-1890.

3. O'Brien JE Jr, Kibiryeva N, Zhou XG, Marshall JA, Lofland GK, Artman M, Chen J, Bittel DC: Noncoding RNA expression in myocardium from infants with tetralogy of Fallot. Circ Cardiovasc Genet 2012, 5(3):279-286.

4. Di Felice V, Zummo G: Tetralogy of Fallot as a Model to Study Cardiac Progenitor Cell Migration and Differentiation During Heart Development. Trends Cardiovas Med 2009, 19(4):130-135.

5. Bruneau BG: The developmental genetics of congenital heart disease. Nature 2008, 451(7181):943-948.

6. Draus JM Jr, Hauck MA, Goetsch M, Austin EH 3rd, Tomita-Mitchell A Mitchell ME: Investigation of somatic NKX2-5 mutations in congenital heart disease. J Med Genet 2009, 46(2):115-122.

7. Salazar M, Consoli F, Villegas V, Caicedo V, Maddaloni V, Daniele P, Caianiello G, Pachon S, Nunez F, Limongelli G, et al: Search of somatic GATA4 and NKX2.5 gene mutations in sporadic septal heart defects. Eur J Med Genet 2011, 54(3):306-309.

8. Olson EN: Gene regulatory networks in the evolution and development of the heart. Science 2006, 313(5795):1922-1927

9. Cheng Z, Lib L, Li Z, Liu M, Yan J, Wang B, Ma X: Two novel HAND1 mutations in Chinese patients with ventricular septal defect. Clin Chim Acta 2012, 413(7-8):675-677.

10. Reamon-Buettner SM, Ciribilli Y, Traverso I, Kuhls B, Inga A, Borlak J: A functional genetic study identifies HAND1 mutations in septation defects of the human heart. Hum Mol Genet 2009, 18(19):3567-3578.

11. Eldadah ZA, Hamosh A, Biery NJ, Montgomery RA, Duke M, Elkins R, Dietz HC: Familial Tetralogy of Fallot caused by mutation in the jagged1 gene. Hum Mol Genet 2001, 10(2):163-169.

12. Bauer RC, Laney AO, Smith R, Gerfen J, Morrissette JJ, Woyciechowski S, Garbarini J, Loomes KM, Krantz ID, Urban Z, et al: Jagged1 (JAG1) mutations in patients with tetralogy of Fallot or pulmonic stenosis. Hum Mutat 2010, 31(5):594-601.

13. Guida V, Ferese R, Rocchetti M, Bonetti M, Sarkozy A, Cecchetti S, Gelmetti $V$, Lepri F, Copetti M, Lamorte G, et al: A variant in the carboxyl-terminus of connexin 40 alters GAP junctions and increases risk for tetralogy of Fallot. Eur J Hum Genet 2013, 21(1):69-75.

14. De Luca A, Sarkozy A, Ferese R, Consoli F, Lepri F, Dentici ML, Vergara P, De Zorzi A, Versacci P, Digilio MC, et al: New mutations in ZFPM2/FOG2 gene in tetralogy of Fallot and double outlet right ventricle. Clin Genet 2011, 80(2):184-190.

15. Stennard FA, Costa MW, Elliott DA, Rankin S, Haast SJ, Lai D, McDonald LP, Niederreither K, Dolle P, Bruneau BG, et al: Cardiac T-box factor Tbx20 directly interacts with Nkx2-5, GATA4, and GATA5 in regulation of gene expression in the developing heart. Dev Biol 2003, 262(2):206-224.

16. Esposito G, Butler TL, Blue GM, Cole AD, Sholler GF, Kirk EP, Grossfeld P, Perryman BM, Harvey RP, Winlaw DS: Somatic mutations in NKX2-5, GATA4, and HAND1 are not a common cause of tetralogy of Fallot or hypoplastic left heart. Am J Med Genet A 2011, 155A(10):2416-2421.

17. Quach N, Goodman MF, Shibata D: In vitro mutation artifacts after formalin fixation and error prone translesion synthesis during PCR. BMC Clin Pathol 2004, 4(1):1.

18. Bittel DC, Butler MG, Kibiryeva N, Marshall JA, Chen J, Lofland GK, O'Brien JE $\mathrm{Jr}$ : Gene expression in cardiac tissues from infants with idiopathic conotruncal defects. BMC Med Genomics 2011, 4:1

19. Pierpont ME, Basson CT, Benson DW Jr, Gelb BD, Giglia TM, Goldmuntz E, McGee G, Sable CA, Srivastava D, Webb CL: Genetic basis for congenital heart defects: current knowledge: a scientific statement from the American Heart Association Congenital Cardiac Defects Committee, Council on Cardiovascular Disease in the Young: endorsed by the American Academy of Pediatrics. Circulation 2007, 115(23):3015-3038

20. Liu L, Li Y, Tollefsbol TO: Gene-environment interactions and epigenetic basis of human diseases. Curr Issues Mol Biol 2008, 10(1-2):25-36.

21. Goll MG, Bestor TH: Eukaryotic cytosine methyltransferases. Annu Rev Biochem 2005, 74:481-514.

22. Jones PA, Baylin SB: The epigenomics of cancer. Cell 2007, 128(4):683-692
23. Vaitkiene $P$, Skiriute $D$, Skauminas $K$, Tamasauskas A: GATA4 and DcR1 methylation in glioblastomas. Diagn Pathol 2013, 8:7.

24. Rahmatpanah FB, Carstens S, Guo J, Sjahputera O, Taylor KH, Duff D, Shi H, Davis JW, Hooshmand SI, Chitma-Matsiga R, et al: Differential DNA methylation patterns of small B-cell lymphoma subclasses with different clinical behavior. Leukemia 2006, 20(10):1855-1862.

25. Feng Q, Balasubramanian A, Hawes SE, Toure P, Sow PS, Dem A, Dembele $B$, Critchlow CW, Xi L, Lu H, et al: Detection of hypermethylated genes in women with and without cervical neoplasia. J Natl Cancer Inst 2005, 97 (4):273-282.

26. Ehrich $M$, Turner J, Gibbs $P$, Lipton L, Giovanneti $M$, Cantor $C$, van den Boom D: Cytosine methylation profiling of cancer cell lines. Proc Natl Acad Sci U S A 2008, 105(12):4844-4849.

27. Sheng W, Wang H, Ma X, Qian Y, Zhang P, Wu Y, Zheng F, Chen L, Huang G, Ma D: LINE-1 methylation status and its association with tetralogy of fallot in infants. BMC Med Genomics 2012, 5:20.

28. Reed K, Poulin ML, Yan L, Parissenti AM: Comparison of bisulfite sequencing PCR with pyrosequencing for measuring differences in DNA methylation. Anal Biochem 2010, 397(1):96-106.

29. Ollikainen M, Smith KR, Joo EJ, Ng HK, Andronikos R, Novakovic B, Abdul Aziz NK, Carlin JB, Morley R, Saffery R, et al: DNA methylation analysis of multiple tissues from newborn twins reveals both genetic and intrauterine components to variation in the human neonatal epigenome. Hum Mol Genet 2010, 19(21):4176-4188.

30. Ohtani K, Dimmeler S: Epigenetic regulation of cardiovascular differentiation. Cardiovasc Res 2011, 90(3):404-412

31. Yagi S, Hirabayashi K, Sato S, Li W, Takahashi Y, Hirakawa T, Wu G, Hattori N, Ohgane J, Tanaka S, et al: DNA methylation profile of tissue-dependent and differentially methylated regions (T-DMRs) in mouse promoter regions demonstrating tissue-specific gene expression. Genome Res 2008, 18(12):1969-1978

32. Zhang T, Wang L, Wang F, Guan J, Le J, Wu LH, Zou JZ, Zhao HZ, Pei LJ, Zheng $X Y$ : Relation between hypomethylation of long interspersed nucleotide elements and risk of neural tube defects. Am J Clin Nutr 2010, 91(5):1359-1367.

33. Thompson RF, Suzuki M, Lau KW, Greally JM: A pipeline for the quantitative analysis of CG dinucleotide methylation using mass spectrometry. Bioinformatics 2009, 25(17):2164-2170.

34. Marin-Garcia J: Advances in molecular genetics of congenital heart disease. Rev Esp Cardiol 2009, 62(3):242-245

35. Wang J, Lu Y, Chen H, Yin M, Yu T, Fu Q: Investigation of somatic NKX2-5, GATA4 and HAND1 mutations in patients with tetralogy of Fallot. Pathology 2011, 43(4):322-326.

doi:10.1186/1755-8794-6-46

Cite this article as: Sheng et al: DNA methylation status of NKX2-5, GATA4 and HAND1 in patients with tetralogy of fallot. BMC Medical Genomics 2013 6:46.

\section{Submit your next manuscript to BioMed Central and take full advantage of:}

- Convenient online submission

- Thorough peer review

- No space constraints or color figure charges

- Immediate publication on acceptance

- Inclusion in PubMed, CAS, Scopus and Google Scholar

- Research which is freely available for redistribution 